\title{
Minimally invasive spine surgery in lumbar spondylodiscitis: a retrospective single-center analysis of 67 cases
}

\author{
Anja Tschugg ${ }^{1} \cdot$ Sebastian Hartmann $^{1} \cdot$ Sara Lener $^{1} \cdot$ Andreas Rietzler $^{2} \cdot$ \\ Neururer Sabrina ${ }^{3} \cdot$ Claudius Thomé $^{1}$
}

Received: 29 January 2017/Revised: 5 May 2017/Accepted: 6 June 2017/Published online: 12 June 2017

(c) The Author(s) 2017. This article is an open access publication

\begin{abstract}
Background Minimally invasive surgical techniques have been developed to minimize tissue damage, reduce narcotic requirements, decrease blood loss, and, therefore, potentially avoid prolonged immobilization. Thus, the purpose of the present retrospective study was to assess the safety and efficacy of a minimally invasive posterior approach with transforaminal lumbar interbody debridement and fusion plus pedicle screw fixation in lumbar spondylodiscitis in comparison to an open surgical approach. Furthermore, treatment decisions based on the patients preoperative condition were analyzed.

Methods 67 patients with lumbar spondylodiscitis treated at our department were included in this retrospective analysis. The patients were categorized into two groups based on the surgical procedure: group (MIS) minimally invasive lumbar spinal fusion $(n=19)$; group (OPEN) open lumbar spinal fusion $(n=48)$. Evaluation included radiological parameters on magnetic resonance imaging (MRI), laboratory values, and clinical outcome.

Results Preoperative MRI showed higher rates of paraspinal abscess ( 35.5 vs. $5.6 \% ; p=0.016$ ) and multilocular location in the OPEN group (20 vs. $0 \%, p=0.014$ ). Overall pain at discharge was less in the MIS group: NRS $2.4 \pm 1$ vs. NRS $1.6 \pm 1 \quad(p=0.036)$. The duration of
\end{abstract}

Anja Tschugg

anja.tschugg@i-med.ac.at

1 Department of Neurosurgery, Medical University Innsbruck, Anichstr. 35, 6020 Innsbruck, Austria

2 Department of Neuroradiology, Medical University Innsbruck, Innsbruck, Austria

3 Department of Medical Statistics and Health Economics, Medical University Innsbruck, Innsbruck, Austria hospital stay was longer in the OPEN than the MIS group $(19.1 \pm 12$ days vs. $13.7 \pm 5$ days, $p=0.018)$.

Conclusion The open technique is effective in all varieties of spondylodiscitis inclusive in epidural abscess formation. MIS can be applied safely and effectively as well in selected cases, even with epidural abscess.

Keywords Minimally invasive spine surgery ·

Spondylodiscitis - Epidural abscess - Spinal infection .

Transforaminal lumbar interbody fusion

\section{Introduction}

The incidence of spondylodiscitis is increasing due to an aging population, immune-compromising diseases, and frequent spine procedures [1]. It is a life-threatening and serious disease with high mortality rates [2]. Surgery for lumbar spondylodiscitis is indicated for neurological deficits, instability, deformity, medically intractable pain, or disease progression [3, 4]. The surgical goal in lumbar spondylodiscitis is the debridement of infection, identification and reduction of pathogens, stabilization of deformed and instable segments, and decompression of neural structures [3-7].

Lumbar pedicle screw fixation with transforaminal lumbar interbody fusion (TLIF) is an established, safe, and effective surgical technique in patients with infective spondylodiscitis. It provides an adequate surgical debridement and enables immediate stabilization, if necessary [6]. Nevertheless, open pedicle screw fixation with TLIF is an invasive surgical intervention that may be associated with several risks [8]. Therefore, minimally invasive techniques have been developed for degenerative diseases and have been applied in infected cases to 
minimize tissue damage, reduce narcotic requirements, decrease blood loss, and, therefore, potentially avoid prolonged immobilization [9-12]. This is of great interest especially in spondylodiscitis patients who frequently suffer from severe comorbidities.

Thus, the purpose of the present retrospective study was to detect the safety and efficacy of a minimal invasive posterior approach with transforaminal lumbar interbody debridement and fusion plus pedicle screw fixation in lumbar spondylodiscitis in comparison to an open surgical approach. Based on these data, treatment decisions tailored to the patients general condition were analyzed.

\section{Materials and methods}

A retrospective review of patients who underwent surgical treatment for lumbar spondylodiscitis at our department was performed. 67 consecutive patients were identified between 2000 and 2016 and their data were retrospectively investigated. The patients were categorized into two groups based on the operative procedure: group (MIS) minimally invasive lumbar spinal fusion; group (OPEN) open lumbar spinal fusion. The two groups were compared at the time of initial diagnosis, the day of admission, on the third postoperative day, before discharge, and after 3 month followup. Data were collected using the patientś health records and magnetic resonance imaging (MRI). MRI was read by an independent neuroradiologist, blinded to the patients clinical data. C-reactive protein (CRP), hemoglobin, and complete white cell count were analyzed routinely. Patients with neurological deficits, progressive pain, or progression on MRI despite conservative treatment underwent surgical treatment. Each treatment option was accompanied by broad spectrum antibiotic therapy or antibiotics according to the antiobiogram when available. Patients were treated with intravenous broad spectrum antibiotics for 2-6 weeks followed by oral antibiotics for a total period of 3 months.

\section{Operative procedure}

The techniques described below have originally been developed for degenerative diseases and have been applied in infected cases. The operations were performed by two experienced surgeons. In the minimal invasive setting, two parasagittal skin incisions of approximately $3 \mathrm{~cm}$ were applied. The pedicle screws were positioned via a transmuscular route. In the open technique, a midline skin incision of approximately $10 \mathrm{~cm}$ was performed and the paravertebral muscles were mobilized laterally. In both cases, a cage was inserted via a one-sided facetectomy in a transforaminal lumbar interbody fusion (TLIF) technique. When performing a minimally invasive TLIF, the skin incision was placed at least $4 \mathrm{~cm}$ lateral to the midline to allow good access to the contralateral disc space and an expandable tubular retractor system was used (Fig. 1) $[13,14]$.

\section{Statistical analysis}

All patients with complete initial data were considered for inclusion in the retrospective analysis. All values are expressed as mean $\pm \mathrm{SD}$. The Kolmogorov-Smirnov test was used for testing for normal distribution. The unpaired Students $t$ test and Mann-Whitney $U$ test were used to analyze differences in clinical and demographic characteristics and in clinical outcome variables. Frequencies were compared by Chi-square and Fisher's exact tests. A $p$ value $<0.05$ was considered statistically significant. All statistical evaluations were performed with SPSS Version 21.0 (IBM Corp. Released 2012. IBM SPSS Statistics for Windows, Version 21.0, NY: IBM Corp.). Figures were designed using GraphPad Prism (version 5.0 for Mac OS X, GraphPad Software, La Jolla California USA, http:// www.graphpad.com).

\section{Results}

The demographic details and patients' characteristics are presented in Table 1. Sixty-seven consecutive patients who underwent lumbar fusion surgery for lumbar spondylodiscitis have been identified between 2000 and 2016 and their data were retrospectively investigated. Thereby, the proportion of patients who underwent minimal invasive surgery was $28.4 \%$ (group MIS, $n=19$ ) versus $71.6 \%$ (group OPEN, $n=48$ ) with open surgical procedures. Altogether, $43(64.2 \%)$ patients were female. The most common ASA score was ASA $3^{\circ}$ in the OPEN group, whereas ASA $2^{\circ}$ and $3^{\circ}$ have been mostly detected in MIS group $(p>0.05)$. There have been no differences in comorbidities or addiction between groups $(p>0.05)$.

Preoperative MRI showed higher rates of paraspinal abscess (35.5 vs. $5.6 \%, p=0.016)$ and multilocular location in the OPEN group (20 vs. $0 \%, p=0.014$ ). There were trends that epidural abscesses and paraspinal enhancement occurred more frequently in the OPEN group than in the MIS group, but this was not statistically significant $(p>0.05)$. No differences could be revealed in disc or vertebral bone destruction between the two groups $(p>0.05)$ (Fig. 2). Mean disc height of the affected discs was $5.8 \pm 2 \mathrm{~mm}$ in the OPEN group and $5.3 \pm 2 \mathrm{~mm}$ in the MIS group $(p>0.05)$.

$1.8 \pm 1$ lumbar levels in the OPEN group and $1.3 \pm 0.8$ levels in the MIS group were fused $(p>0.05)$. The intraoperative amount of blood transfusion was higher in the 

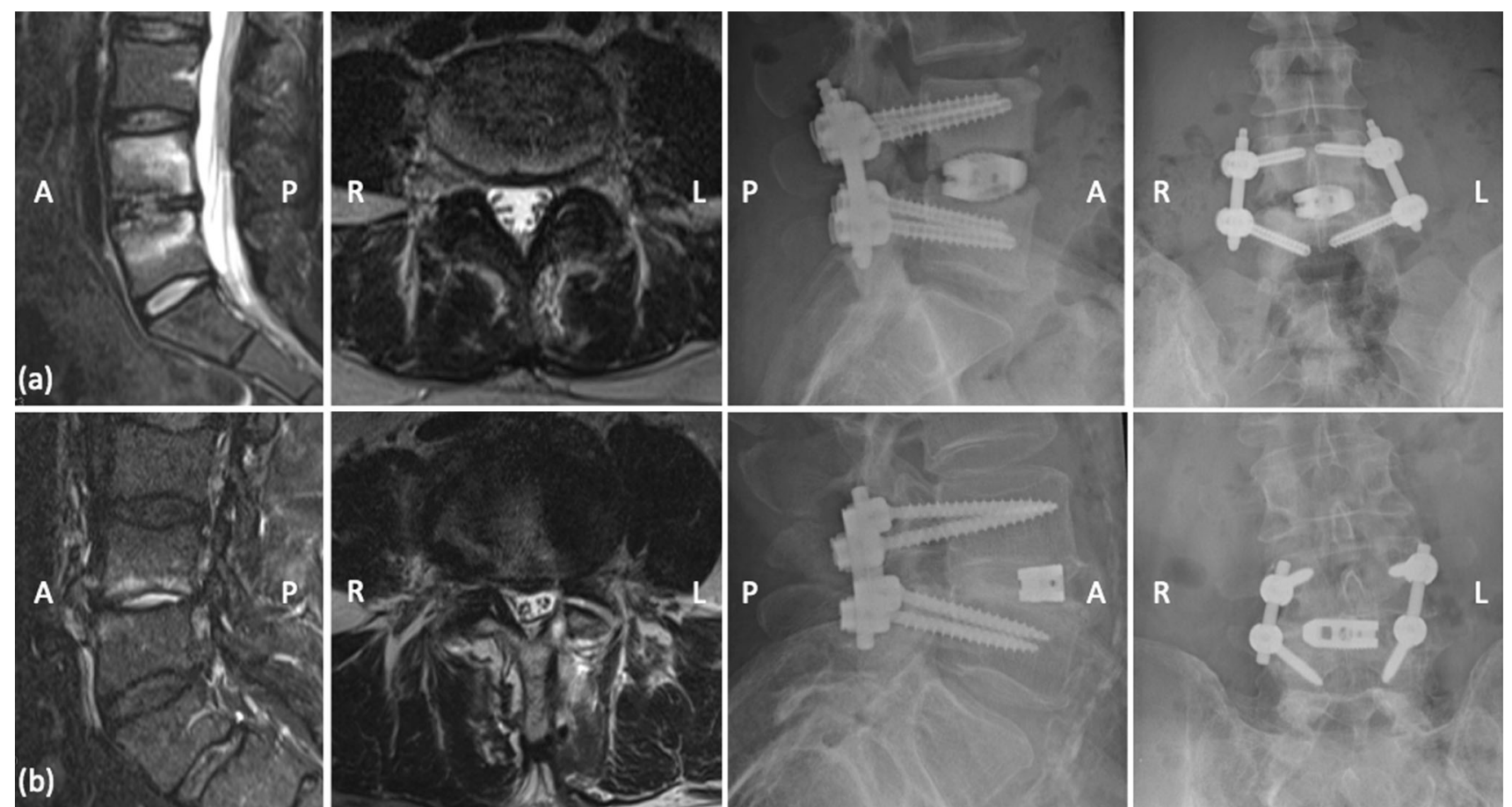

Fig. 1 MRI and X-ray imaging. Preoperative sagittal and axial T2weighted MRI sequences and postoperative sagittal and coronary $\mathrm{X}$-ray imaging (from left to right) in a minimal invasive pedicle screw

fixation in comparison to $\mathbf{b}$ the open pedicle screw instrumentation in patients with lumbar spondylodiscitis. $A$ anterior, $p$ posterior, $L$ left, $R$ right

OPEN group $(472.3 \pm 555$ vs. $110.5 \pm 205 \mathrm{ml}$, $p=0.015)$. No differences occurred in duration of surgery between the two groups: OPEN $208.8 \pm 86 \mathrm{~min}$ vs. MIS $173.4 \pm 71 \min (p>0.05)$.

CRP and leucocytes differed significantly preoperatively ( $p<0.05$; Fig. 3). Overall pain at discharge was less in the MIS group: NRS $2.4 \pm 1$ vs. NRS $1.6 \pm 1(p=0.036)$. No differences were present after 3 months follow-up $(p>0.05) .51 .1 \%$ in the OPEN vs. $18.8 \%$ in the MIS cohort suffered from a motor deficit at first diagnosis ( $p=0.026$ ). For more details, see Fig. 4. The hospital stay was longer in the OPEN than the MIS group (19.1 \pm 12 vs. $13.7 \pm 5$ days, $p=0.018$ ). No differences occurred in postoperative complications (Table 2).

\section{Discussion}

The authors present a retrospective study comparing patients with lumbar spondylodiscitis that underwent either a minimally invasive or an open transpedicular lumbar spinal fusion. Motor deficits were more common as well as higher inflammatory blood values could be detected in patients in which the open approach was performed. Patients who underwent minimally invasive spine surgery showed less pain at discharge. After 3 months of follow-up, improvement of pain and motor function was equally distributed in both groups.

Lumbar pedicle screw fixation with transforaminal lumbar interbody fusion (TLIF) is an established, safe, and effective surgical technique in patients with infective spondylodiscitis. It provides an adequate surgical debridement and enables immediate stabilization, if necessary [6]. However, a TLIF is still associated with major risks, especially in elderly or immunocompromised patients and patients with multiple comorbidities [8]. Thus, minimally invasive surgical techniques have been developed to avoid and reduce tissue damage. The treatment goal of MIS in lumbar spondylodiscitis is to decrease muscle crush injuries during retraction and to avoid disruption of the osseotendinous complex of the paraspinal muscles. In addition, although MIS uses a smaller skin incision, adequate surgical debridement is necessary to achieve good clinical results [6, 12].

An appropriate access to the spinal canal was attained in both surgical techniques. When a medial facet resection was done to decompress the ipsilateral side of the spinal canal, a hemilaminectomy could be performed. Furthermore, if the tube was angled toward the contralateral side, the contralateral side of the spinal canal could also be reached [14]. Epidural abscess formation was detected on preoperative MRI in both groups and could be removed adequately by the surgical technique mentioned above. 
Table 1 Demographic details in open (OPEN) and minimally invasive (MIS) treated patients with lumbar spondylodiscitis

\begin{tabular}{|c|c|c|c|}
\hline & Group OPEN $(n=48)$ & Group MIS $(n=19)$ & $p$ value \\
\hline Age (in years) & $64.4 \pm 12$ & $63.9 \pm 12$ & n.s. \\
\hline Female gender, $n(\%)$ & $32(66.7)$ & $11(57.9)$ & n.s. \\
\hline BMI & $27.4 \pm 6$ & $24.9 \pm 3$ & n.s. \\
\hline \multicolumn{4}{|l|}{ ASA score } \\
\hline $1^{\circ}, n(\%)$ & $2(4.2)$ & $2(10.5)$ & n.s. \\
\hline $2^{\circ}, n(\%)$ & $15(31.3)$ & $8(42.1)$ & n.s. \\
\hline $3^{\circ}, n(\%)$ & $29(60.4)$ & $8(42.1)$ & n.s. \\
\hline $4^{\circ}, n(\%)$ & $2(4.2)$ & $1(5.3)$ & n.s. \\
\hline Duration of first diagnosis until surgery (in days) & $20.2 \pm 44$ & $36.6 \pm 45$ & 0.012 \\
\hline Duration of hospital stay (in days) & $19.1 \pm 12$ & $13.7 \pm 5$ & 0.018 \\
\hline Spondylodiscitis following lumbar surgery, $n(\%)$ & $27(56.3)$ & $7(36.8)$ & n.s. \\
\hline Primary conservative treatment before surgery, $n(\%)$ & $30(62.5)$ & $16(84.2)$ & n.s. \\
\hline \multicolumn{4}{|l|}{ Comorbidities } \\
\hline Dental disease, $n(\%)$ & $1(2.1)$ & $1(5.3)$ & n.s. \\
\hline Renal failure, $n(\%)$ & $3(6.3)$ & $2(10.5)$ & n.s. \\
\hline Diabetes, $n(\%)$ & $8(16.7)$ & $3(15.8)$ & n.s. \\
\hline Heart diseases, $n(\%)$ & $14(29.2)$ & $8(42.1)$ & n.s. \\
\hline Hepatopathy, $n(\%)$ & $7(14.6)$ & $1(5.3)$ & n.s. \\
\hline \multicolumn{4}{|l|}{ Addiction } \\
\hline Smoking, $n(\%)$ & $13(27.7)$ & $4(22.2)$ & n.s. \\
\hline Cigarettes (per day) & $5.0 \pm 10$ & $6.5 \pm 14$ & n.s. \\
\hline Alcohol, $n(\%)$ & $15(31.2)$ & $5(26.3)$ & n.s. \\
\hline Drug abuse, $n(\%)$ & $10(20.8)$ & $1(5.3)$ & n.s. \\
\hline
\end{tabular}

$n$ number of patients, $p$ statistical significance, n.s. not statistically significant, $B M I$ body mass index
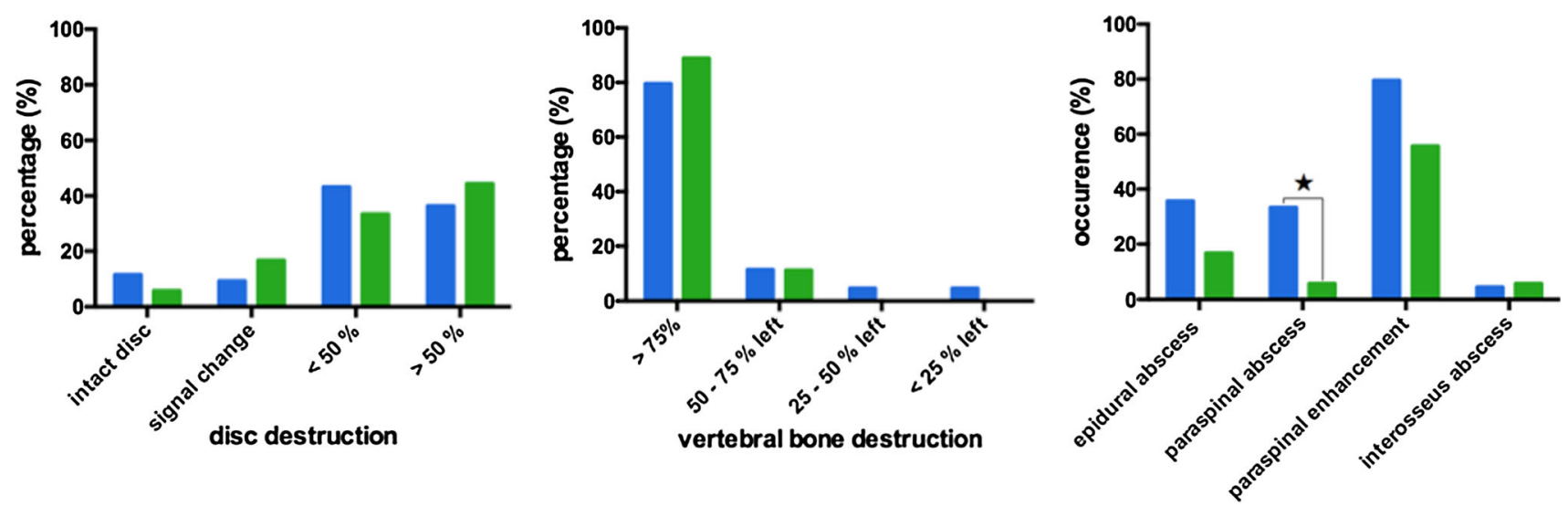

OPEN $\square$ MIS $\star p<0.05$

Fig. 2 Preoperative magnetic resonance imaging findings. Group (MIS) minimally invasive lumbar spinal fusion, group (OPEN) open lumbar spinal fusion. $p$ significance

According to the recent published literature [15], no differences were found in the duration of surgery in our study population. These results are likely attributed to the local surgical expertise. Minimal invasive surgery for spondylodiscitis in our department is exclusively undertaken by neurosurgeons who are already experienced with tubular surgical techniques for lumbar decompression. Nevertheless, in the MIS group, the percentage of misplaced screws was 5.3\%, which may due to the loss of visual anatomic landmarks and reduced tactile feedback [16]. 

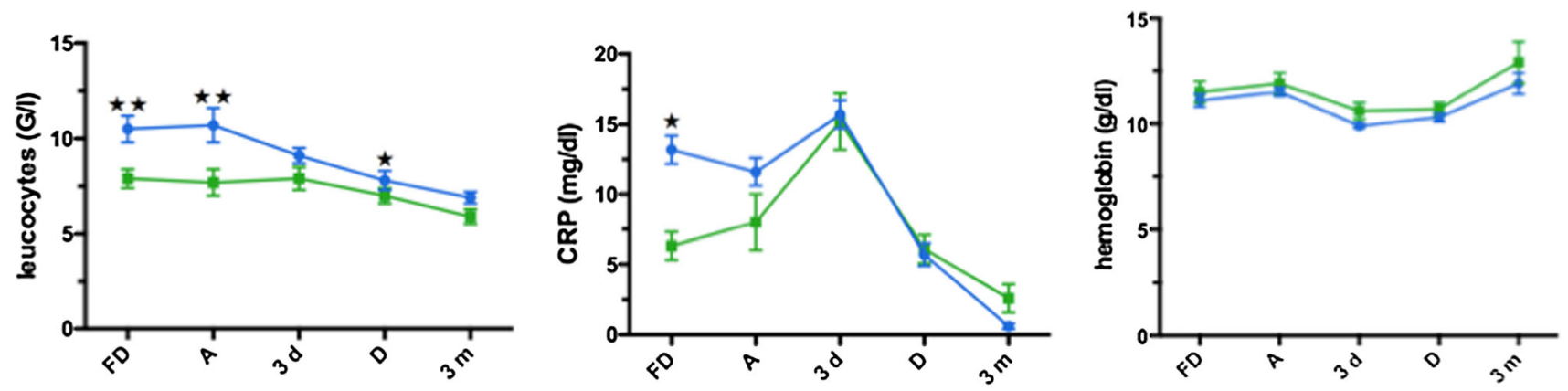

MIS

OPEN $\star: p<0.05$

$<0.005$

Fig. 3 Inflammatory blood values in open (OPEN) and minimally invasive (MIS) treated patients with lumbar spondylodiscitis. $p$ significance, $C R P$ C-reactive protein, $F D$ first diagnosis, $A$ admission, $d$ days, $D$ discharge, $m$ month

Fig. 4 Motor deficits and numeric rating scale for pain in open (OPEN) and minimally invasive (MIS) treated patients with lumbar spondylodiscitis. $p$ significance, $F D$ first diagnosis, $A$ admission, $d$ days, $D$ discharge, $m$ month

\section{ङั 100}

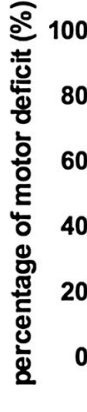

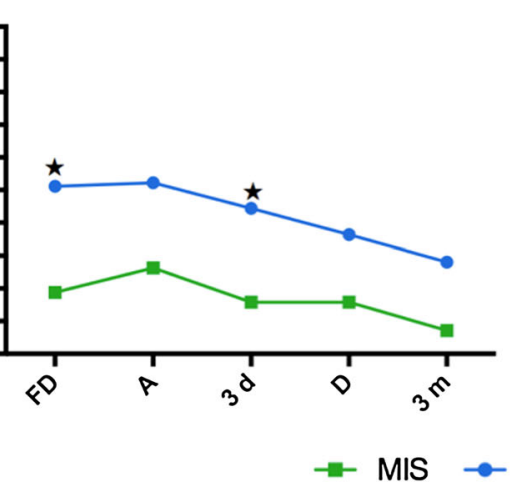

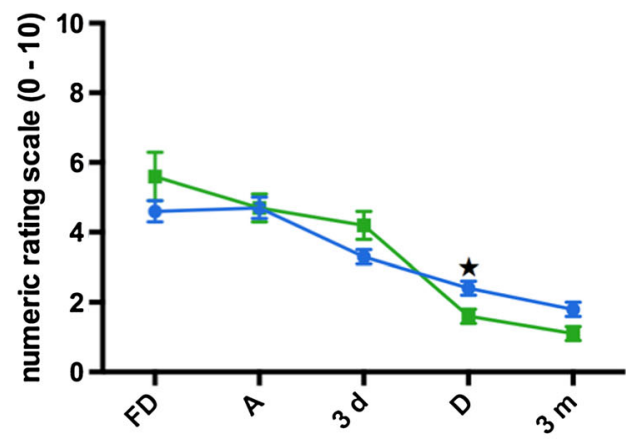

OPEN $\star: p<0.05$
Table 2 Postoperative complications in open (OPEN) and minimally invasive (MIS) treated patients with lumbar spondylodiscitis

\begin{tabular}{lcll}
\hline & OPEN $(\%)$ & MIS $(\%)$ & $p$ value \\
\hline Sepsis & 2.1 & 0 & n.s. \\
Screw misplacement & 0 & 5.3 & n.s. \\
Wound infection & 10.4 & 5.3 & n.s. \\
Pulmonal embolism & 2.1 & 0 & n.s. \\
Death & 2.1 & 0 & n.s. \\
\hline
\end{tabular}

$p$ statistical significance, $n . s$. not statistically significant

Patients who underwent MIS showed significantly lower pain scores at discharge compared to patients undergoing the open surgical technique, whereas no differences could be revealed 3 months after surgery. The differences between groups found proved to be statistically significant, but do not seem to be clinically relevant according to the recent literature on the minimal clinical important difference of NRS [17].

Furthermore, titanium cages were preferably used in our study group to achieve adequate stability and to avoid pseudoarthrosis. Titanium has been successfully proven to provide sufficient resistance to microbial adherence and showed good radiological results in pyogenic spondylodiscitis [18, 19]. Poly-ether-ether-ketone (PEEK) cages as biocompatible alternatives to titanium implants have been used in spinal fusion surgery, but still have been an issue of debate in pyogenic spondylodiscitis. Recent published data could show favorable results on the application of PEEK cages, but prospective randomized controlled trials are lacking [20, 21].

There is still controversy regarding the indication of MIS in lumbar spondylodiscitis. Decision-making is predominantly based on the individual surgeońs preference and skill. In our study group, the open procedure was favored especially in patients with abscess formation and in the presence of a motor deficit, whereas comorbidities or age did not seem to influence a surgeon's treatment decision. In severe cases with high inflammatory values, surgery was performed earlier in the infectious cascade. In addition, the delay from the first diagnosis until surgery was significantly shorter in the OPEN group. These facts may be contributed to the evidently sicker patients in this group. On the contrary, the levels to be treated did not seem to influence the decision for an open or minimal invasive surgery.

Additional studies with greater patient numbers are required for definitive confirmation. However, beside the low number of patients, we have to mention the 
retrospective character of our study results and the short follow-up period as further limitations.

\section{Conclusion}

Both the MIS and the open technique were used in this retrospective study. The patients who were treated by the open approach were evidently sicker. Less postoperative pain before discharge was detected in the MIS group. The open technique is effective in all varieties of spondylodiscitis inclusive in epidural abscess formation. MIS can be applied safely and effectively as well in selected cases, even with epidural abscess. Nevertheless, prospective randomized controlled clinical trials will be mandatory to develop evidence-based treatment recommendations.

Acknowledgements Open access funding provided by University of Innsbruck and Medical University of Innsbruck.

\section{Compliance with ethical standards}

Conflict of interest None of the authors has any conflict of interest in connection with the study.

Ethical approval According to the local institutional review board, for this type of retrospective study, ethics approval is not required.

Funding The study did not receive any external funding.

Informed consent According to the local institutional review board, for this type of retrospective study, informed consent is not required.

Open Access This article is distributed under the terms of the Creative Commons Attribution 4.0 International License (http://crea tivecommons.org/licenses/by/4.0/), which permits unrestricted use, distribution, and reproduction in any medium, provided you give appropriate credit to the original author(s) and the source, provide a link to the Creative Commons license, and indicate if changes were made.

\section{References}

1. Tsiodras S, Falagas ME (2006) Clinical assessment and medical treatment of spine infections. Clin Orthop Relat Res 444:38-50

2. Kehrer M, Pedersen C, Jensen TG, Hallas J, Lassen AT (2015) Increased short- and long-term mortality among patients with infectious spondylodiscitis compared with a reference population. Spine J 15:1233-1240

3. Grados F, Lescure FX, Senneville E, Flipo RM, Schmit JL, Fardellone P (2007) Suggestions for managing pyogenic (nontuberculous) discitis in adults. Jt Bone Spine 74:133-139

4. Stüer C, Stoffel M, Hecker J, Ringel F, Meyer B (2013) A staged treatment algorithm for spinal infections. J Neurol Surg A Cent Eur Neurosurg 74:87-95

5. Ghobrial GM, Beygi S, Viereck MJ, Maulucci CM, Sharan A, Heller J et al (2014) Timing in the surgical evacuation of spinal epidural abscesses. Neurosurg Focus 37:E1

6. Lu ML, Niu CC, Tsai TT, Fu TS, Chen LH, Chen WJ (2015) Transforaminal lumbar interbody debridement and fusion for the treatment of infective spondylodiscitis in the lumbar spine. Eur Spine J 24:555-560

7. Keric N, Eum DJ, Afghanyar F, Rachwal-Czyzewicz I, Renovanz M, Conrad J et al (2016) Evaluation of surgical strategy of conventional vs. percutaneous robot-assisted spinal trans-pedicular instrumentation in spondylodiscitis. J Robot Surg 11:17-25

8. Carreon LY, Puno RM, Dimar JR, Glassman SD, Johnson JR (2003) Perioperative complications of posterior lumbar decompression and arthrodesis in older adults. J Bone Jt Surg Am 85(11):2089-2092

9. Rieger B, Jiang H, Ruess D, Reinshagen C, Molcanyi M, Zivcak J et al (2016) First clinical results of minimally invasive vector lumbar interbody fusion (MIS-VLIF) in spondylodiscitis and concomitant osteoporosis: a technical note. Eur Spine J. doi:10. 1007/s00586-016-4928-z (epub 2016 Dec 27)

10. Turel MK, Kerolus M, Deutsch H (2017) The role of minimally invasive spine surgery in the management of pyogenic spinal discitis. J Craniovertebr Junction Spine 8:39-43

11. Viezens L, Schaefer C, Helmers R, Vettorazzi E, Schroeder M, Hansen-Algenstaedt N (2017) Spontaneous pyogenic spondylodiscitis in the thoracic or lumbar spine: a retrospective cohort study comparing the safety and efficacy of minimally invasive and open surgery over a nine-year period. World Neurosurg. doi:10.1016/j.wneu.2017.02.129 (epub 2017 Mar 9)

12. Khan NR, Clark AJ, Lee SL, Venable GT, Rossi NB, Foley KT (2015) Surgical outcomes for minimally invasive vs open transforaminal lumbar interbody fusion: an updated systematic review and meta-analysis. Neurosurgery 77:847-874 (discussion 874)

13. Mobbs RJ, Phan K, Malham G, Seex K, Rao PJ (2015) Lumbar interbody fusion: techniques, indications and comparison of interbody fusion options including PLIF, TLIF, MI-TLIF, OLIF/ ATP, LLIF and ALIF. J Spine Surg 1:2-18

14. German JW, Foley KT (2005) Minimal access surgical techniques in the management of the painful lumbar motion segment. Spine (Phila Pa 1976) 30:S52-S59

15. Phan K, Rao PJ, Kam AC, Mobbs RJ (2015) Minimally invasive versus open transforaminal lumbar interbody fusion for treatment of degenerative lumbar disease: systematic review and metaanalysis. Eur Spine J 24:1017-1030

16. Ravi B, Zahrai A, Rampersaud R (2011) Clinical accuracy of computer-assisted two-dimensional fluoroscopy for the percutaneous placement of lumbosacral pedicle screws. Spine (Phila Pa 1976) 36:84-91

17. Copay AG, Glassman SD, Subach BR, Berven S, Schuler TC, Carreon LY (2008) Minimum clinically important difference in lumbar spine surgery patients: a choice of methods using the Oswestry Disability Index, Medical Outcomes Study questionnaire Short Form 36, and pain scales. Spine J 8:968-974

18. Robinson Y, Tschoeke SK, Finke T, Kayser R, Ertel W, Heyde CE (2008) Successful treatment of spondylodiscitis using titanium cages: a 3 -year follow-up of 22 consecutive patients. Acta Orthop 79:660-664

19. Robinson Y, Tschoeke SK, Kayser R, Boehm H, Heyde CE (2009) Reconstruction of large defects in vertebral osteomyelitis with expandable titanium cages. Int Orthop 33:745-749

20. Tschöke SK, Fuchs H, Schmidt O, Gulow J, von der Hoeh NH, Heyde CE (2015) Single-stage debridement and spinal fusion using PEEK cages through a posterior approach for eradication of lumbar pyogenic spondylodiscitis: a safe treatment strategy for a detrimental condition. Patient Saf Surg 9:35

21. Schomacher M, Finger T, Koeppen D, Süss O, Vajkoczy P, Kroppenstedt $S$ et al (2014) Application of titanium and polyetheretherketone cages in the treatment of pyogenic spondylodiscitis. Clin Neurol Neurosurg 127:65-70 